\title{
Magnetic Field and Gravity Effects on Peristaltic Transport of a Jeffrey Fluid in an Asymmetric Channel
}

\author{
A. M. Abd-Alla, ${ }^{1,2}$ S. M. Abo-Dahab, ${ }^{1,3}$ and Maram M. Albalawi ${ }^{1}$ \\ ${ }^{1}$ Mathematics Department, Faculty of Science, Taif University, Taif 888, Saudi Arabia \\ ${ }^{2}$ Mathematics Department, Faculty of Science, Sohag University, Sohag, Egypt \\ ${ }^{3}$ Mathematics Department, Faculty of Science, SVU, Qena 83523, Egypt \\ Correspondence should be addressed to A. M. Abd-Alla; mohmrr@yahoo.com
}

Received 12 January 2014; Accepted 19 February 2014; Published 13 April 2014

Academic Editor: Juntao Sun

Copyright (C) 2014 A. M. Abd-Alla et al. This is an open access article distributed under the Creative Commons Attribution License, which permits unrestricted use, distribution, and reproduction in any medium, provided the original work is properly cited.

\begin{abstract}
In this paper, the peristaltic flow of a Jeffrey fluid in an asymmetric channel has been investigated. Mathematical modeling is carried out by utilizing long wavelength and low Reynolds number assumptions. Closed form expressions for the pressure gradient, pressure rise, stream function, axial velocity, and shear stress on the channel walls have been computed numerically. Effects of the Hartmann number, the ratio of relaxation to retardation times, time-mean flow, the phase angle and the gravity field on the pressure gradient, pressure rise, streamline, axial velocity, and shear stress are discussed in detail and shown graphically. The results indicate that the effect of Hartmann number, ratio of relaxation to retardation times, time-mean flow, phase angle, and gravity field are very pronounced in the peristaltic transport phenomena. Comparison was made with the results obtained in the presence and absence of magnetic field and gravity field.
\end{abstract}

\section{Introduction}

Peristaltic pumping has been the object of scientific and engineering research in recent years. The word peristaltic comes from a Greek word "Peristaltikos" which means clasping and compressing. The peristaltic transport is travelling contraction wave along a tube-like structure, and it results physiologically from neuron-muscular properties of any tubular smooth muscle. Peristaltic motion of blood (or other fluid) in animal or human bodies have been considered by many authors. It is an important mechanism for transporting blood, where the cross section of the artery is contracted or expanded periodically by the propagation of progressive wave. It plays an indispensable role in transporting many physiological fluids in the body in various situations such as urine transport from the kidney to the bladder through the ureter, transport of spermatozoa in the ducts efferents of the male reproductive tract, and the movement of the ovum in the flipping tubes.

A variety of complex theological fluids can easily be transported from one place to another with a special type of pumping known as peristaltic pumping. This pumping principle is called peristalsis. The mechanism includes involuntary periodic contraction followed by relaxation or expansion of the ducts that the fluids move through; this leads to the rise of pressure gradient that eventually pushes the fluid forward.

The study of the peristaltic transport of a fluid in the presence of an external magnetic field and rotation is of great importance with regard to certain problems involving the movement of conductive physiological fluids, for example, blood and saline water. Pandey and Chaube [1] investigated an analytical study of the MHD flow of a micropolar fluid through a porous medium induced by sinusoidal peristaltic waves traveling down the channel wall. The magnetohydrodynamic flow of a micropolar fluid in a circular cylindrical tube has been investigated by Wang et al. [2]. Nadeem and Akram [3] studied the analytical and numerical treatment of peristaltic flows in viscous and non-Newtonian fluids. Vajravelu et al. [4] studied the influence of heat transfer on peristaltic transport of a Jeffrey fluid in a vertical porous stratum. Hayat et al. [5] discussed the influence of compliant 
wall properties and heat transfer on the peristaltic flow of an incompressible viscous fluid in a curved channel. Bhargava et al. [6] have studied finite element study of nonlinear twodimensional deoxygenated biomagnetic micropolar flow. Ali et al. [7] discussed the peristaltic motion of a non-Newtonian fluid in a channel having compliant boundaries. Abd-Alla et al. [8] studied the effects of rotation and magnetic field on nonlinear peristaltic flow of second-order fluid in an asymmetric channel through a porous medium. Hayat et al. [9] analyzed the effect of an induced magnetic field on the peristaltic flow of an incompressible Carreau fluid in an asymmetric channel. Pandey et al. [10] were concerned with the theoretical study of two-dimensional peristaltic flow of power-law fluids in three layers with different viscosities. Jiménez-Lozano and Sen [11] investigated the streamline patterns and their local and global bifurcations in a twodimensional planar and axisymmetric peristaltic flow for an incompressible Newtonian fluid. Hayat et al. [12] analyzed the effect of an induced magnetic field on the peristaltic flow of an incompressible Carreau fluid in an asymmetric channel. Srinivas and Kothandapani [13] investigated the effects of heat and mass transfer on peristaltic transport in a porous space with compliant walls. Gad [14] discussed the effect of hall currents on the interaction of pulsatile and peristaltic transport induced flows of a particle fluid suspension. Abd-Alla et al. [15] investigated the peristaltic flow in a tube with an endoscope subjected to a magnetic field. Hayat and Noreen [16] discussed the influence of an induced magnetic field on the peristaltic flow of an incompressible fourth grade fluid in a symmetric channel with heat transfer. Nadeem and Akbar [17] investigated the peristaltic flow of an incompressible MHD Newtonian fluid in a vertical annulus. Nadeem and Akbar [18] investigated the deals with the peristaltic motion of an incompressible non-Newtonian fluid in a nonuniform tube for long wavelength. Nadeem et al. [19] studied the concentrates on the heat transfer characteristics and endoscope effects for the peristaltic flow of a thirdorder fluid. Srinivas et al. [20] studied the effects of both wall slip conditions and heat transfer on the peristaltic flow of MHD Newtonian fluid in a porous channel with elastic wall properties. Abd-Alla et al. [21] investigated the effect of the rotation, magnetic field, and initial stress on peristaltic motion of micropolar fluid. Mahmoud et al. [22] discussed the effect of the rotation on wave motion through cylindrical bore in a micropolar porous medium. The dynamic behavior of a wet long bone that has been modeled as a piezoelectric hollow cylinder of crystal class 6 is investigated by Abd-Alla et al. [23]. Akram and Nadeem [24] discussed the peristaltic motion of a two-dimensional Jeffrey fluid in an asymmetric channel under the effects of the induced magnetic field and heat transfer.

Recently, Rathod and Mahadev [25] investigated the effect of heat transfer and magnetic field on the peristaltic transport of Jeffrey fluid in an inclined channel taking slip condition into account. S. N. Reddy and G. V. Reddy [26] studied the effect of sleep on the peristaltic flow of a Jeffrey fluid through a porous medium in an asymmetric channel under the assumptions of long wavelength and low Reynolds number.
The aim of this paper is to study the effect of magnetic field and gravity field on peristaltic motion of Jeffrey type and is electrically conducting in asymmetric channel. Here the governing equations are nonlinear in nature; we used infinitely long wavelength assumption to obtain linearized system of coupled differential equations which are then solved analytically. Results have been discussed for pressure gradient, pressure rise, streamline and axial velocity to observe the Hartmann number, the ratio of relaxation to retardation times, time-mean flow, the phase angle, and the gravity field effect. The numerical result displayed by figures and the physical meaning is explained. The results and discussions presented in this study may be helpful to further understand MHD peristaltic motion for non-Newtonian fluids in an asymmetric channel.

\section{Formulation of the Problem}

Let us consider the peristaltic transport of an incompressible viscous fluid in a two-dimensional channel of width $d_{1}+d_{2}$. The channel walls are inclined at angles $\alpha$. The flow is induced by sinusoidal wave trains propagating with constant speed $c$ along the channel walls.

The geometry of the wall surfaces is

$$
\begin{aligned}
& \overline{h_{1}}(\bar{X}, \bar{t})=d_{1}+a_{1} \cos \left[\frac{2 \pi}{\lambda}(\bar{X}-c \bar{t}]\right. \text { at upper wall, } \\
& \bar{h}_{2}(\bar{X}, \bar{t})=-d_{2}-b_{1} \cos \left[\frac{2 \pi}{\lambda}(\bar{X}-c \bar{t})+\phi\right] \text { at lower wall, }
\end{aligned}
$$

where $a_{1}$ and $b_{1}$ are the types of amplitude of the waves, $\lambda$ is the wavelength, $c$ is the wave speed, $\phi(0 \leq \phi \leq \pi)$ is the phase difference, $\phi=0$ corresponds to symmetric channel with waves out of phase, and $\phi=\pi$ indicates that the waves are in phase, and further $a_{1}, b_{1}, d_{1}, d_{2}$, and $\phi$ satisfy the condition

$$
a_{1}^{2}+b_{1}^{2}+2 a_{1} b_{1} \cos \phi \preccurlyeq\left(d_{1}+d_{2}\right)^{2}
$$

The Cauchy $(\bar{T})$ and extra $(\bar{S})$ stress tensors take the following form

$$
\begin{gathered}
\bar{T}=-\bar{p} \bar{I}+\bar{S}, \\
\bar{S}=\frac{\mu}{1+\lambda_{1}}\left(\overline{\dot{\gamma}}+\lambda_{2} \overline{\ddot{\gamma}}\right),
\end{gathered}
$$

where $\bar{p}$ is the pressure, $\bar{I}$ is the identity tensor, $\lambda_{1}$ is the ratio of relaxation to retardation times, $\lambda_{2}$ is the retardation time, and $\dot{\gamma}$ is the shear rate. 
In laboratory frame, the following set of pertinent field equations governing the flow are

$$
\begin{aligned}
\rho\left[\frac{\partial}{\partial t}+\bar{U} \frac{\partial}{\partial \bar{X}}+\bar{V} \frac{\partial}{\partial \bar{Y}}\right] \bar{U}= & -\frac{\partial \bar{p}}{\partial \bar{X}}+\frac{\partial}{\partial \bar{X}}\left(\bar{S}_{\bar{X} \bar{X}}\right) \\
& +\frac{\partial}{\partial \bar{Y}}\left(\bar{S}_{\bar{X} \bar{Y}}\right)-\sigma B_{0}^{2} \bar{U}+\rho g \sin \alpha \\
\rho\left[\frac{\partial}{\partial t}+\bar{U} \frac{\partial}{\partial \bar{X}}+\bar{V} \frac{\partial}{\partial \bar{Y}}\right] \bar{V}= & -\frac{\partial \bar{p}}{\partial \bar{Y}}+\frac{\partial}{\partial \bar{X}}\left(\bar{S}_{\bar{X} \bar{Y}}\right) \\
& +\frac{\partial}{\partial \bar{Y}}\left(\bar{S}_{\bar{Y} Y}\right)-\rho g \cos \alpha
\end{aligned}
$$

and the continuity equation takes the form

$$
\frac{\partial \bar{U}}{\partial \bar{X}}+\frac{\partial \bar{V}}{\partial \bar{Y}}=0
$$

where $\bar{U}$ and $\bar{V}$ are the velocity components in the laboratory frame $(\bar{X}, \bar{Y}), \rho$ is the density, $g$ is the earth gravity acceleration, $B_{0}$ is the magnetic induction, and $\sigma$ is the electrical conductivity of the fluid.

The flow is inherently unsteady in the laboratory frame $(\bar{X}, \bar{Y})$. However, the flow becomes steady in a wave frame $(\bar{x}, \bar{y})$ moving away from the laboratory frame with speed $c$ in the direction of propagation of the wave. Taking $u$ and $v$ the velocity components in $x$ and $y$ directions, the transformation from the laboratory frame to the wave frame is given by

$$
\begin{gathered}
\bar{x}=\bar{X}-c \bar{t}, \quad \bar{y}=\bar{Y}, \quad \bar{u}=\bar{U}-c, \\
\bar{v}=\bar{V}, \quad \bar{P}(\bar{x})=\bar{P}(\bar{X}, \bar{t}),
\end{gathered}
$$

where $\bar{u}$ and $\bar{v}$ are the velocity components in the wave frame $(\bar{x}, \bar{y}), \bar{p}$ and $\bar{P}$ are pressure in wave and fixed frame of references, respectively.

The appropriate nondimensional variables for the flow are defined as

$$
\begin{gathered}
x=\frac{2 \pi \bar{x}}{\lambda}, \quad y=\frac{\bar{y}}{d_{1}}, \quad u=\frac{\bar{u}}{c}, \\
v=\frac{\bar{v}}{c \bar{\delta}}, \quad \delta=\frac{2 \pi d_{1}}{\lambda}, \\
p=\frac{2 \pi d_{1}^{2} \bar{p}}{\mu c \lambda}, \quad t=\frac{2 \pi c \bar{t}}{\lambda}, \quad h_{1}=\frac{\overline{h_{1}}}{d_{1}}, \\
h_{2}=\frac{\overline{h_{2}}}{d_{1}}, \quad S=\frac{d_{1} \bar{S}}{\mu c}, \quad \Omega=\frac{a_{1}}{a_{2}}, \quad b=\frac{b_{1}}{b_{2}}, \quad \Omega=\frac{d_{2}}{\mu}, \quad a=
\end{gathered}
$$

and the stream function is

$$
u=\frac{\partial \psi}{\partial y}, \quad v=-\delta \frac{\partial \psi}{\partial x}
$$

Using (6), (7a), and (7b) into (3)-(4) and eliminating pressure by cross differentiation, we get

$$
\begin{aligned}
\delta \operatorname{Re}\left[\left(\frac{\partial \psi}{\partial y} \frac{\partial}{\partial x}-\frac{\partial \psi}{\partial x} \frac{\partial}{\partial y}\right) \nabla^{2} \psi\right]= & {\left[\left(\frac{\partial^{2}}{\partial y^{2}}-\delta^{2} \frac{\partial^{2}}{\partial x^{2}}\right) s_{x y}\right] } \\
& +\delta\left[\frac{\partial^{2}}{\partial x \partial y}\left(s_{x x}-s_{x y}\right)\right] \\
& +M^{2} \frac{\partial^{2} \psi}{\partial y^{2}}+\operatorname{Re} \frac{g d_{1}}{c^{2}} \sin \alpha
\end{aligned}
$$

in which

$$
\begin{array}{r}
s_{x x}=\frac{2 \delta}{1+\lambda_{1}}\left[1+\frac{\delta \lambda_{2} c}{d_{1}}\left(\frac{\partial \psi}{\partial y} \frac{\partial}{\partial x}+\frac{\partial \psi}{\partial x} \frac{\partial}{\partial y}\right)\right] \frac{\partial^{2} \psi}{\partial x \partial y} \\
s_{x y}=\frac{1}{1+\lambda_{1}}\left[1+\frac{\delta \lambda_{2} c}{d_{1}}\left(\frac{\partial \psi}{\partial y} \frac{\partial}{\partial x}-\frac{\partial \psi}{\partial x} \frac{\partial}{\partial y}\right)\right] \\
\times\left(\frac{\partial^{2} \psi}{\partial y^{2}}-\delta^{2} \frac{\partial^{2} \psi}{\partial x^{2}}\right) \\
s_{y y}=-\frac{2 \delta}{1+\lambda_{1}}\left[1+\frac{\delta \lambda_{2} c}{d_{1}}\left(\frac{\partial \psi}{\partial y} \frac{\partial}{\partial x}-\frac{\partial \psi}{\partial x} \frac{\partial}{\partial y}\right)\right] \frac{\partial^{2} \psi}{\partial x \partial y}
\end{array}
$$

where

$$
\begin{aligned}
& \nabla^{2}=\delta^{2} \frac{\partial^{2} \psi}{\partial x^{2}}+\frac{\partial^{2} \psi}{\partial y^{2}}, \quad \delta=\frac{2 \pi d_{1}}{\lambda}, \\
& \operatorname{Re}=\frac{\rho c d_{1}}{\mu} \text { is the Reynolds number, } \\
& M=\sqrt{\frac{\sigma}{\mu}} B_{0} d_{1} \text { is the Hartmann number. }
\end{aligned}
$$

Using the long wavelength approximation and neglecting the wave number $\delta$ along with low Reynolds number in our analysis, the field equations (8) and (10) now give

$$
\frac{\partial^{2}}{\partial y^{2}}\left[\frac{1}{1+\lambda_{1}} \frac{\partial^{2} \psi}{\partial y^{2}}\right]+M^{2} \frac{\partial^{2} \psi}{\partial y^{2}}+\operatorname{Re} \frac{g d_{1}}{c^{2}} \sin \alpha=0 .
$$

The boundary conditions for the stream functions in the wave frame are

$$
\begin{gathered}
\psi=\frac{q}{2}, \quad h_{1}=1+a \cos [x] \\
\psi=-\frac{q}{2} \quad \text { at } y=h_{2}(x)=-d-b \cos \left[\frac{2 \pi}{\lambda}(x)+\phi\right], \\
\frac{\partial \psi}{\partial y}=-1 \quad \text { at } y=h_{1}, y=h_{2},
\end{gathered}
$$

where $q$ is the flux in the wave frame and $a, b, \phi$, and $d$ satisfy the relation

$$
a^{2}+b^{2}+2 a b \cos \phi \leq(1+d)^{2} \text {. }
$$



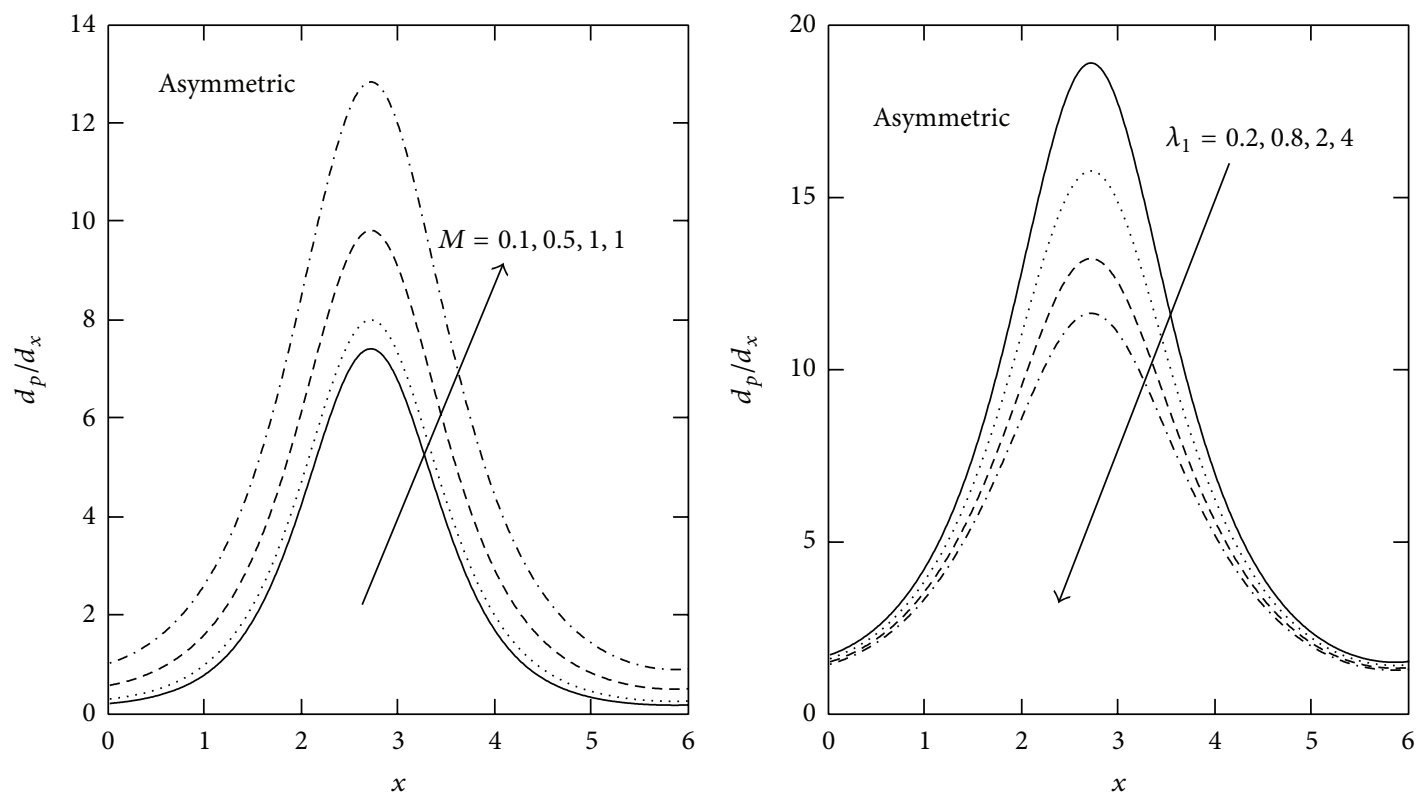

FIGURE 1: Variation of $d p / d x$ with influence of $M$ and $\lambda_{1}$ respect to $x$.
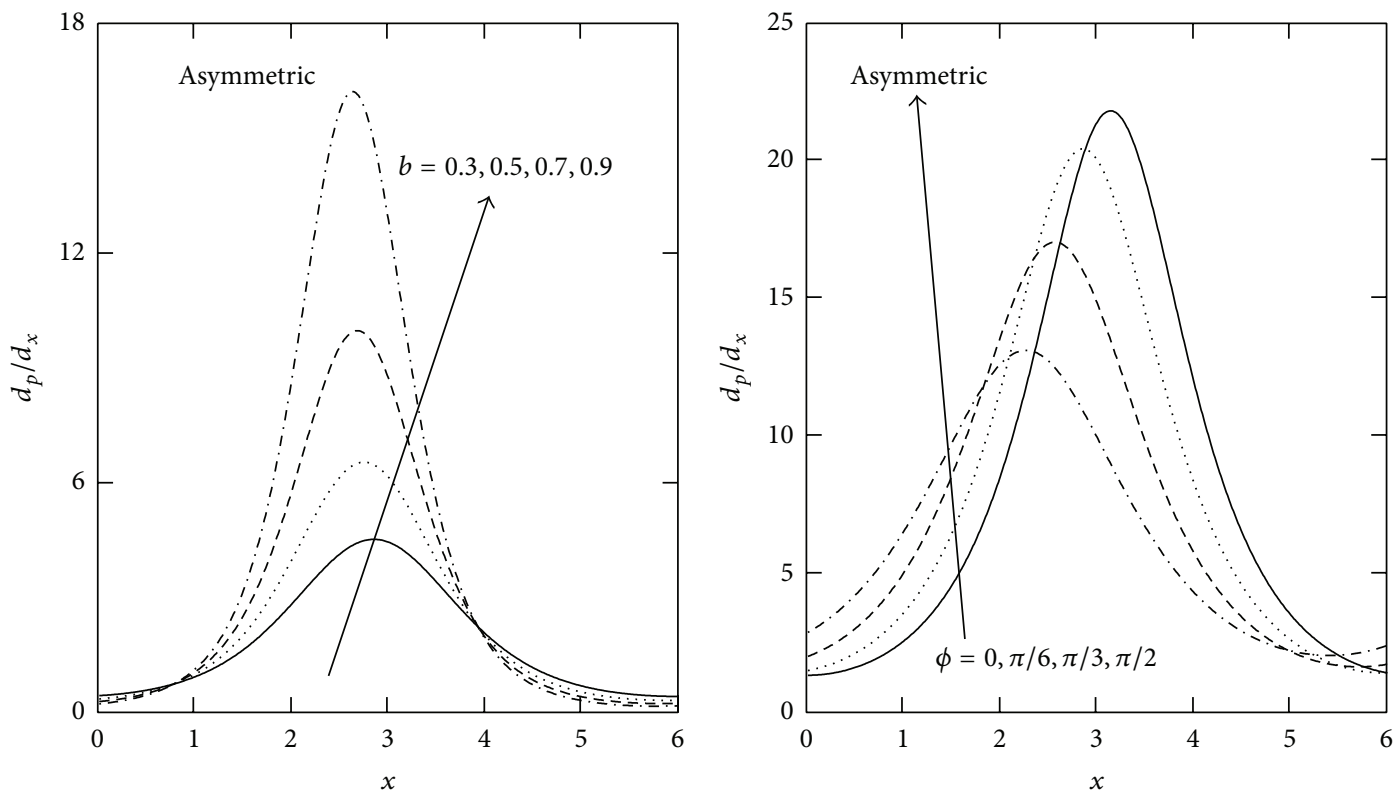

FIgURE 2: Variation of $d p / d x$ with influence of $b$ and $\phi$ respect to $x$.

\section{Solution of the Problem}

The solution of (12) satisfying the corresponding boundary conditions (13) is

$$
\begin{aligned}
\psi= & \frac{\left(h_{1}+h_{2}\right)\left[N q+2 \tanh \left[N\left(h_{1}-h_{2}\right) / 2\right]\right]}{2 N\left(h_{2}-h_{1}\right)+4 \tanh \left[N\left(h_{1}-h_{2}\right) / 2\right]} \\
& +\frac{N q+2 \tanh \left[N\left(h_{1}-h_{2}\right) / 2\right]}{N\left(h_{1}-h_{2}\right)-2 \tanh \left[N\left(h_{1}-h_{2}\right) / 2\right]} y
\end{aligned}
$$

$$
\begin{aligned}
& +\left(\left(q+h_{1}-h_{2}\right) \operatorname{sech}\left[\frac{N\left(h_{1}-h_{2}\right)}{2}\right]\right. \\
& \left.\times \sinh \left[\frac{N\left(h_{1}-h_{2}\right)}{2}\right]\right) \\
& \times\left(N\left(h_{1}-h_{2}\right)-2 \tanh \left[\frac{N\left(h_{1}-h_{2}\right)}{2}\right]\right)^{-1} \\
& \times \cosh (N y)
\end{aligned}
$$



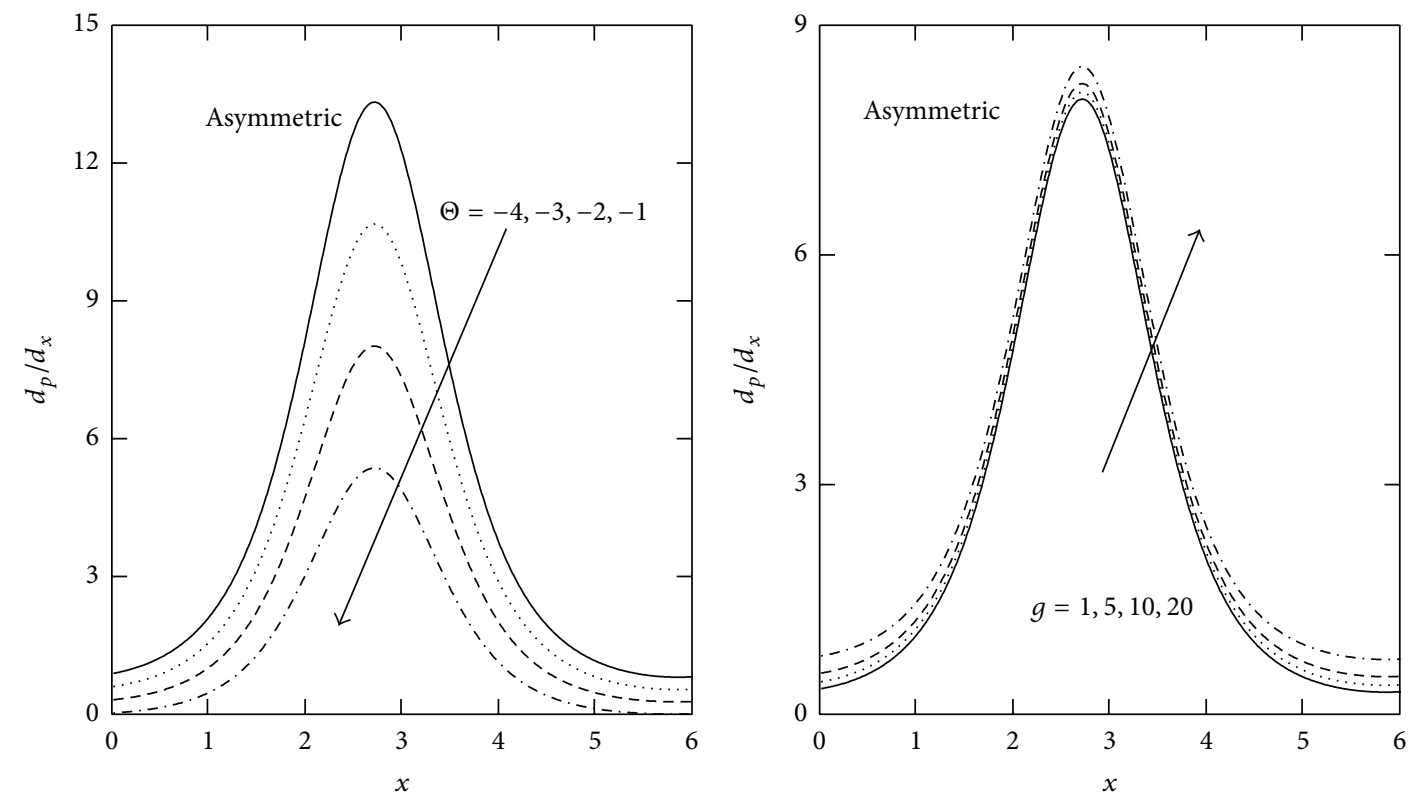

FIGURE 3: Variation of $d p / d x$ with influence of $\Theta$ and $g$ respect to $x$.

$$
\begin{aligned}
& +\left(\left(q+h_{1}-h_{2}\right) \operatorname{sech}\left[\frac{N\left(h_{1}-h_{2}\right)}{2}\right]\right. \\
& \left.\times \cosh \left[\frac{N\left(h_{1}-h_{2}\right)}{2}\right]\right) \\
& \times\left(N\left(h_{1}-h_{2}\right)+2 \tanh \left[\frac{N\left(h_{1}-h_{2}\right)}{2}\right]\right)^{-1} \\
& \times \sinh (N y),
\end{aligned}
$$

where $N^{2}=M^{2}\left(1+\lambda_{1}\right)+\operatorname{Re}\left(g d_{1} / c^{2}\right) \sin \alpha$.

The flux at any axial station in the fixed frame is

$$
Q=\int_{h_{2}}^{h_{1}}\left(\frac{\partial \psi}{\partial y}+1\right) d y=h_{1}-h_{2}+q
$$

The time-mean flow over a period $T$ at a fixed position $\bar{X}$ is defined as

$$
\begin{aligned}
\theta & =\frac{1}{T} \int_{0}^{T} Q d t=\frac{1}{T} \int_{0}^{T}\left(q+h_{1}-h_{2}\right) d t \\
& =q+1+d .
\end{aligned}
$$

The pressure gradient is obtained from the dimensionless momentum equation for the axial velocity as

$$
\begin{aligned}
\frac{d p}{d x}= & \frac{1}{1+\lambda_{1}}\left[\frac{N^{3}\left(q+h_{1}-h_{2}\right)}{N\left(h_{2}-h_{1}\right)+2 \tanh \left[N\left(h_{1}-h_{2}\right) / 2\right]}\right] \\
& +\operatorname{Re} \frac{g d_{1}}{c^{2}} \sin \alpha .
\end{aligned}
$$

The nondimensional expression for the pressure rise per wavelength $\Delta p_{\lambda}$ and frictional forces on the lower $\left(F_{\lambda}^{l}\right)$ and upper $\left(F_{\lambda}^{u}\right)$ walls on the lower are defined as follows:

$$
\begin{aligned}
& \Delta p_{\lambda}=\int_{0}^{2 \pi}\left(\frac{d p}{d x}\right) d x, \\
& F_{\lambda}^{l}=\int_{0}^{1} h_{2}^{2}\left(-\frac{d p}{d x}\right) d x, \\
& F_{\lambda}^{u}=\int_{0}^{1} h_{1}^{2}\left(-\frac{d p}{d x}\right) d x .
\end{aligned}
$$

The nondimensional expression of the shear stress at the upper wall of the channel is reduced to

$$
\begin{aligned}
S_{x y}= & \frac{1}{1+\lambda_{1}} \frac{\partial^{2} \psi}{\partial y^{2}} \\
= & \frac{N^{2}\left(q+h_{1}-h_{2}\right)\left[N\left(h_{1}-h_{2}\right) / 2\right]}{\left(1+\lambda_{1}\right)\left\{N\left(h_{1}-h_{2}\right)-2 \tanh \left[N\left(h_{1}-h_{2}\right) / 2\right]\right\}} \\
& \times\left\{\sinh \left[\frac{N\left(h_{1}-h_{2}\right)}{2}\right] \cosh M h_{1}\right. \\
& \left.\quad-\cosh \left[\frac{N\left(h_{1}-h_{2}\right)}{2}\right] \sinh M h_{1}\right\} .
\end{aligned}
$$

\section{Numerical Results and Discussion}

In order to gain physical insight into the pressure gradient, pressure rise $\Delta p_{\lambda}$, streamline $\psi$, velocity $u$, and shear stress $S_{x y}$ have been discussed by assigning numerical values to the parameter encountered in the problem in which 

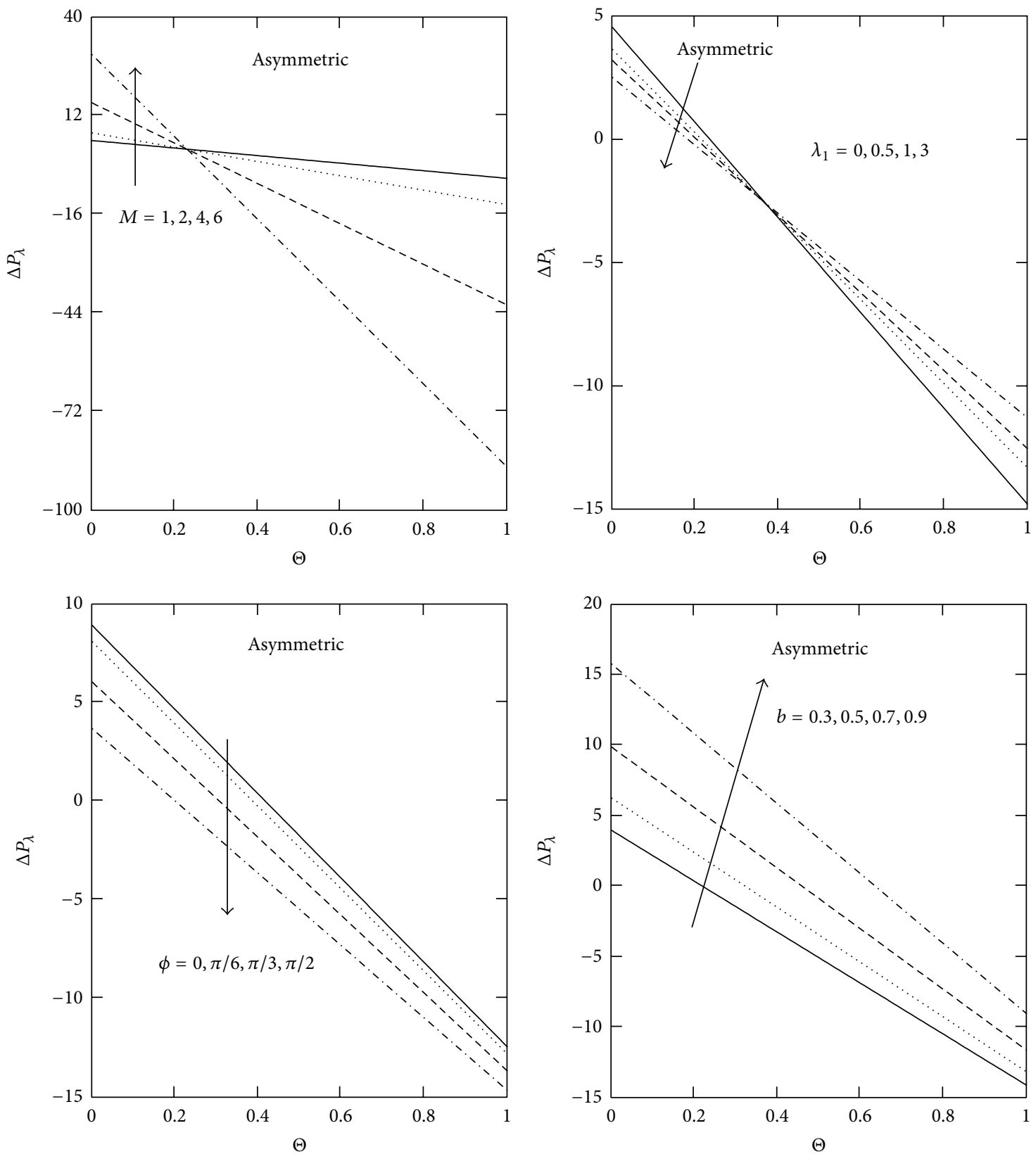

FIgURE 4: Variation of $\Delta P_{\lambda}$ with influence of $M, \lambda_{1}, \phi$, and $b$ with respect to $\Theta$.

the numerical results are displayed with the graphic illustrations. The variations are shown in Figures 1-7, respectively.

Figures 1, 2, and 3 show the variations of the axial pressure gradient $d p / d x$ with respect to the axial $x$ which it has oscillatory behavior in the whole range of the $x$-axis for different values of the Hartmann number $M$, the ratio of relaxation to retardation times $\lambda_{1}$, time-mean flow $\Theta$, the phase angle $\phi$, gravity field $g$, and the nondimensional amplitude of wave $b$ in asymmetric channel. In both figures, it is clear that the pressure gradient has a nonzero value only in a bounded region of space. The effect of the Hartmann number, the ratio of relaxation to retardation times, timemean flow, rotation, gravity field, and the nondimensional amplitude of wave decreases and increases gradually. It is observed that the pressure gradient increases with increasing of the Hartmann number, gravity field, the phase angle, and the nondimensional amplitude of the wave, while it decreases with increasing of the time-mean flow and the ratio of relaxation to retardation times. It is noticed that the axial pressure gradient when compared to the case of asymmetric and symmetric channel takes a large values respect to the small values of $x$ and small values with an increasing of $x$. Moreover, it can be noticed that, on the one hand, in the wider part of the channel $x \in[0,2]$ and $[3,5,6]$, the pressure gradient is relatively small; that is, the flow can easily pass without imposition of a large pressure gradient. On the other 

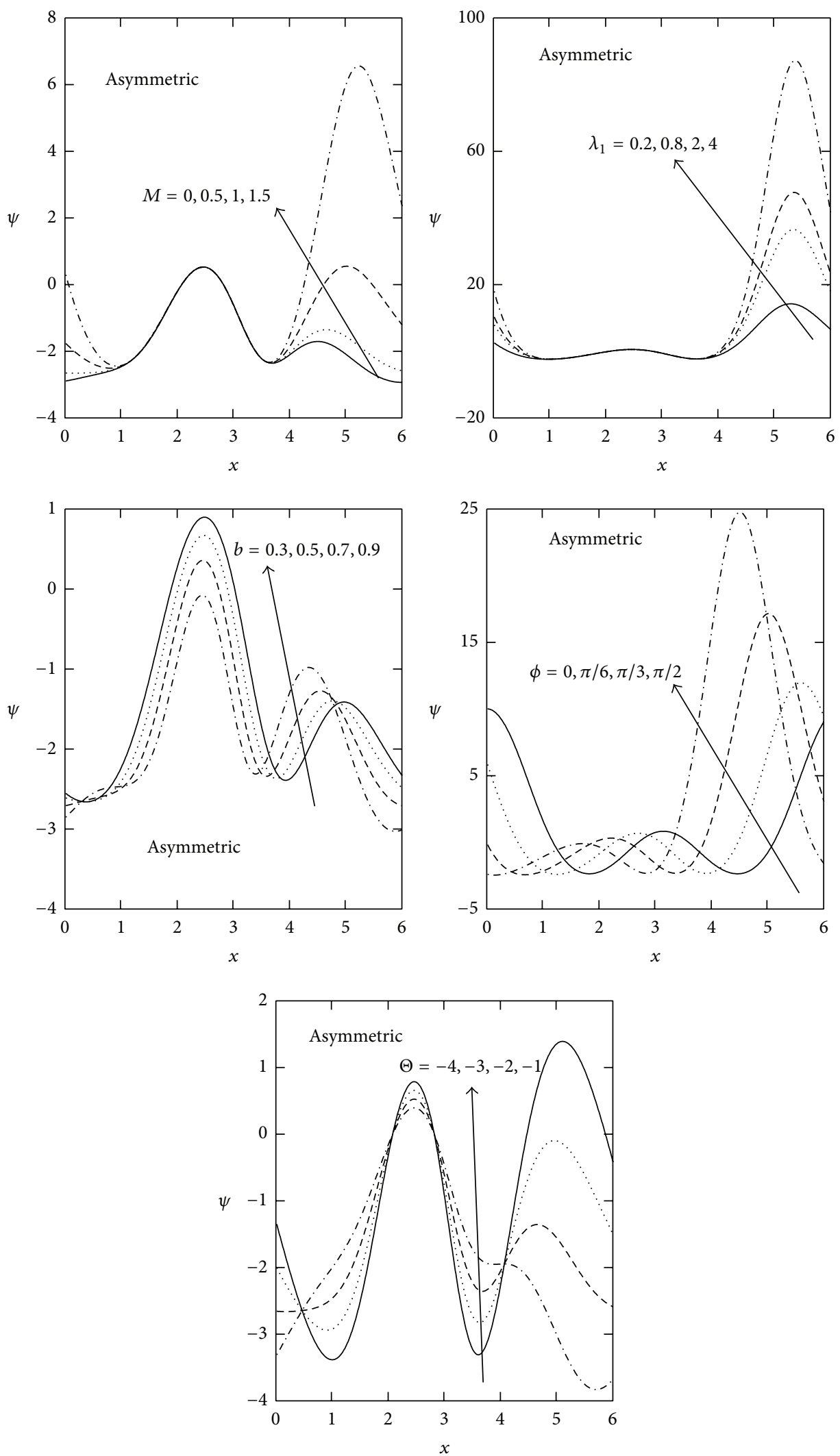

FIgURE 5: Variation of $\psi$ with influence of $M, \lambda_{1}, b, \phi$, and $\Theta$ with respect to $x$. 

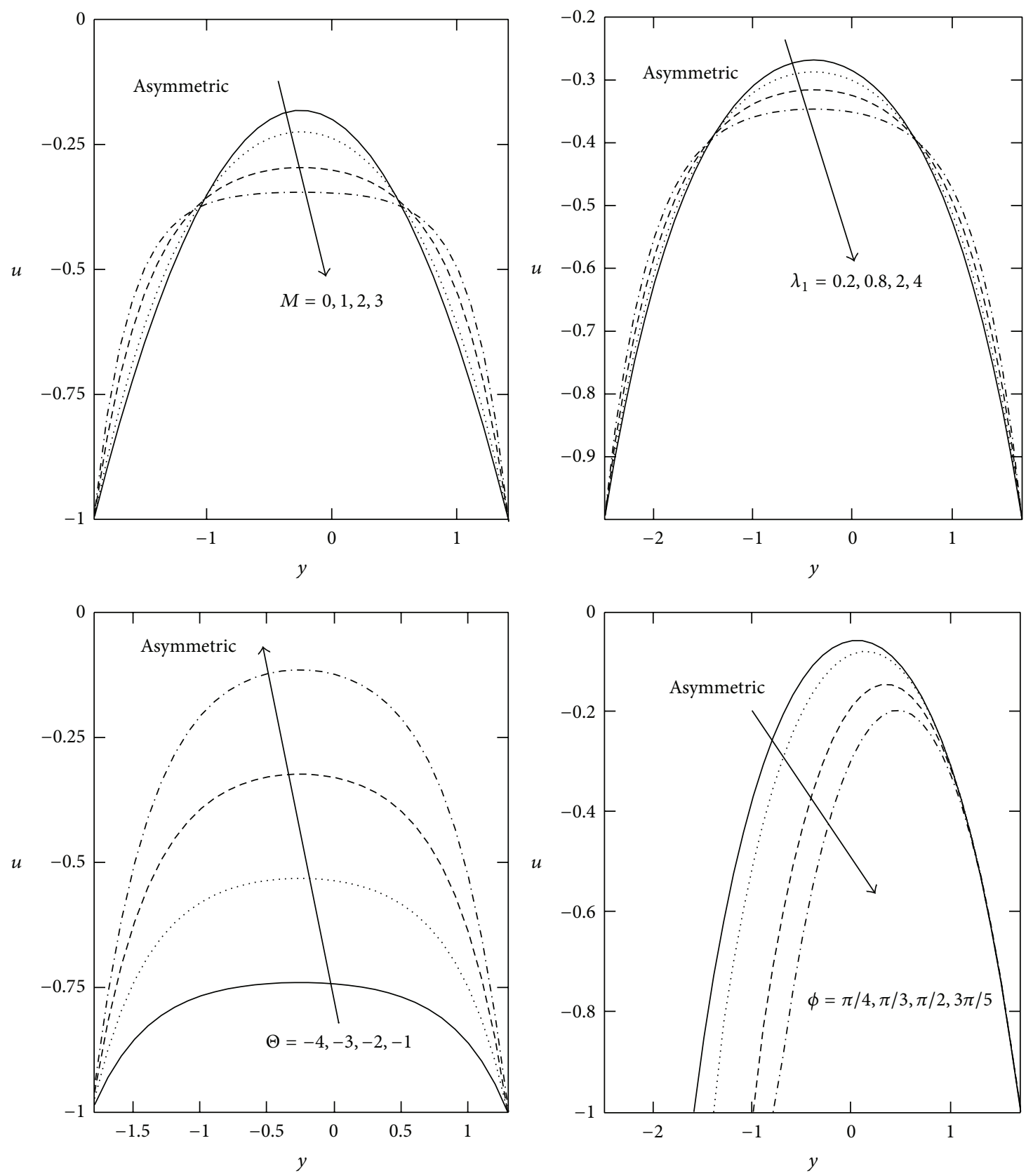

FIGURE 6: Variation of $u$ with influence of $M, \lambda_{1}, \Theta$, and $\phi$ with respect to $y$.

hand, in a narrow part of the channel $x \in[2,3.5]$, a much pressure gradient is required to maintain the flux to pass it especially near $x=2.7$.

Figure 4 shows the variations of the pressure rise $\Delta p_{\lambda}$ with respect to the time-mean flow $\Theta$ for different values of the Hartmann number $M$, the ratio of relaxation to retardation times $\lambda_{1}$, the phase difference $\phi$, and the nondimensional amplitude of wave $b$. In both figures, it is clear that the pressure rise has a nonzero value only in a bounded region of space. It is observed that the pressure rise increases with increasing of the Hartmann number and the nondimensional amplitude of the wave, while it decreases with increasing the time-mean flow, the ratio of relaxation to retardation times, and the phase difference. The graph is sectored so that the upper right-hand quadrant (I) denotes the region of the peristaltic pumping $\left(\Theta>0, \Delta p_{\lambda}>0\right)$. Quadrant (II) is designated as an augmented flow when $\Theta>0, \Delta p_{\lambda}<0$. Quadrant (IV) such that $\Theta<0, \Delta p_{\lambda}>0$ is called retrograde or backward pumping.

Figure 5 shows the variations of the streamlines $\psi$ with respect to the axial $x$ which has oscillatory behavior in the whole range of the $x$-axis for different values of the Hartmann number $M$, the ratio of relaxation to retardation times $\lambda_{1}$, time-mean flow $\Theta$, the phase difference $\phi$, and 

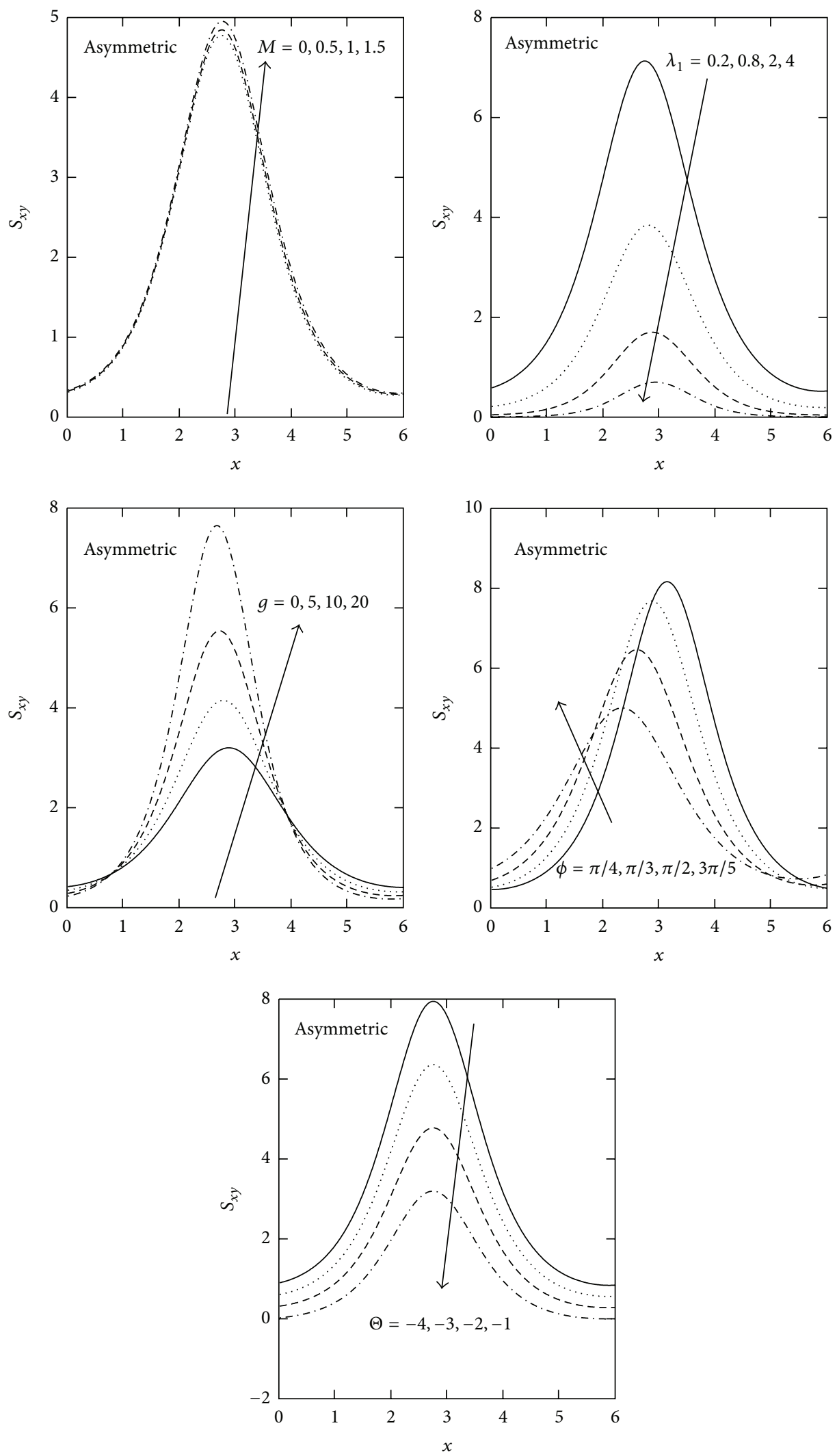

Figure 7: Variation of $S_{x y}$ with influence of $M, \lambda_{1}, \Theta, g$, and $\phi$ with respect to $x$. 
the nondimensional amplitude of wave $b$ in asymmetric channel. In both figures, it is clear that the streamlines have a nonzero value only in a bounded region of space. The effect of the Hartmann number, the ratio of relaxation to retardation times, time-mean flow, the phase angle, and the nondimensional amplitude of wave decreases and increases gradually. It is observed that the streamlines increase with the increasing of the phase angle, the Hartmann number, the time-mean flow, and the ratio of relaxation to retardation times. The streamlines near the channel walls do nearly strictly follow the wall waves, which are mainly engendered by the relative movement of the walls.

Figure 6 shows the variations of the axial velocity $u$ with respect to the axial $y$ which has oscillatory behavior in the whole range of the $y$-axis for different values of the Hartmann number $M$, the ratio of relaxation to retardation times $\lambda_{1}$, time-mean flow $\Theta$, and the phase angle $\phi$ in asymmetric channel. In both figures, it is clear that the axial velocity has a nonzero value only in a bounded region of space. The effect of the Hartmann number, the ratio of relaxation to retardation times, time-mean flow, and the phase angle decreases and increases gradually. It is observed that the axial velocity increases with increasing of the time-mean flow, the Hartmann number, and the ratio of relaxation to retardation, while it decreases with increasing of the phase angle.

Figure 7 displays that the variations of the value of axial shear stress $S_{x y}$ with respect to the axial $x$ has oscillatory behavior may be due to peristalsis in the whole range of the $x$-axis for different values of the Hartmann number $M$, the ratio of relaxation to retardation times $\lambda_{1}$, timemean flow $\Theta$, the phase angle $\phi$, and the gravity field $g$ in asymmetric channel. In both figures, it is clear that the value of shear stress has a nonzero value only in a bounded region of space. The effect of the Hartmann number, the ratio of relaxation to retardation times, time-mean flow, rotation, the phase difference, and the phase angle decreases and increases gradually. It is observed that the shear stress increases with increasing of the Hartmann number, gravity field, and the phase angle, while it decreases with increasing of the ratio of relaxation to retardation times and time-mean flow. Moreover the values of shear stress are larger in case of a Jeffery fluid when compared with Newtonian fluid.

\section{Conclusion}

Due to the complicated nature of the governing equations of the pertinent field equations governing the peristaltic transport of Jeffery fluid, the work done in this field is unfortunately limited in number. The method used in this study is quite successful in dealing with such problems. This method gives exact solutions in the peristaltic transport without any assumed restrictions on the actual physical quantities that appear in the governing equations of the problem considered. Important phenomena are observed in all these computations.

(i) It was found that, for large values of the Hartmann number $M$, the ratio of relaxation to retardation times $\lambda_{1}$, time-mean flow $\Theta$, the phase angle $\phi$, and the gravity field $g$ in asymmetric channel, the solution has been obtained in the context of the peristaltic transport of fluid.

(ii) By comparing Figures 1-7 for the peristaltic transport of fluid with figures without magnetic field and gravity field, it was found that it has the same behavior in the same field.

(iii) The results presented in this paper should prove useful for researchers in science and engineering, as well as for those working on the development of fluid mechanics. The study of the phenomenon of the Hartmann number, the ratio of relaxation to retardation times, time-mean flow, the phase angle, and the gravity field in asymmetric channel influence and operations is also used to improve the conditions of peristaltic motion.

\section{Conflict of Interests}

The authors declare that there is no conflict of interests regarding the publication of this paper.

\section{References}

[1] S. K. Pandey and M. K. Chaube, "Peristaltic flow of a micropolar fluid through a porous medium in the presence of an external magnetic field," Communications in Nonlinear Science and Numerical Simulation, vol. 16, no. 9, pp. 3591-3601, 2011.

[2] Y. Wang, N. Ali, T. Hayat, and M. Oberlack, "Peristaltic motion of a magnetohydrodynamic micropolar fluid in a tube," Applied Mathematical Modelling, vol. 35, no. 8, pp. 3737-3750, 2011.

[3] S. Nadeem and S. Akram, "Peristaltic flow of a Williamson fluid in an asymmetric channel," Communications in Nonlinear Science and Numerical Simulation, vol. 15, no. 7, pp. 1705-1716, 2010.

[4] K. Vajravelu, S. Sreenadh, and P. Lakshminarayana, “The influence of heat transfer on peristaltic transport of a Jeffrey fluid in a vertical porous stratum," Communications in Nonlinear Science and Numerical Simulation, vol. 16, no. 8, pp. 3107-3125, 2011.

[5] T. Hayat, M. Javed, and A. A. Hendi, "Peristaltic transport of viscous fluid in a curved channel with compliant walls," International Journal of Heat and Mass Transfer, vol. 54, no. 7-8, pp. 1615-1621, 2011.

[6] R. Bhargava, O. A. Bég, S. Sharma, and J. Zueco, "Finite element study of nonlinear two-dimensional deoxygenated biomagnetic micropolar flow," Communications in Nonlinear Science and Numerical Simulation, vol. 15, no. 5, pp. 1210-1223, 2010.

[7] N. Ali, T. Hayat, and S. Asghar, "Peristaltic flow of a Maxwell fluid in a channel with compliant walls," Chaos, Solitons and Fractals, vol. 39, no. 1, pp. 407-416, 2009.

[8] A. M. Abd-Alla, S. M. Abo-Dahaba, and H. D. El-Shahrany, "Effects of rotation and magnetic field on nonlinear peristaltic flow of second-order fluid in an asymmetric channel through a porous medium," Chinese Physics B, vol. 22, pp. 325-352, 2013.

[9] T. Hayat, N. Saleem, and N. Ali, "Effect of induced magnetic field on peristaltic transport of a Carreau fluid," Communications in Nonlinear Science and Numerical Simulation, vol. 15, no. 9, pp. 2407-2423, 2010.

[10] S. K. Pandey, M. K. Chaube, and D. Tripathi, "Peristaltic transport of multilayered power-law fluids with distinct viscosities: 
a mathematical model for intestinal flows," Journal of Theoretical Biology, vol. 278, pp. 11-19, 2011.

[11] J. Jiménez-Lozano and M. Sen, "Streamline topologies of twodimensional peristaltic flow and their bifurcations," Chemical Engineering and Processing: Process Intensification, vol. 49, no. 7, pp. 704-715, 2010.

[12] T. Hayat, N. Saleem, S. Asghar, M. Shabab Alhothuali, and A. Alhomaidan, "Influence of induced magnetic field and heat transfer on peristaltic transport of a Carreau fluid," Communications in Nonlinear Science and Numerical Simulation, vol. 16, no. 9, pp. 3559-3577, 2011.

[13] S. Srinivas and M. Kothandapani, "The influence of heat and mass transfer on MHD peristaltic flow through a porous space with compliant walls," Applied Mathematics and Computation, vol. 213, no. 1, pp. 197-208, 2009.

[14] N. S. Gad, "Effect of Hall currents on interaction of pulsatile and peristaltic transport induced flows of a particle-fluid suspension," Applied Mathematics and Computation, vol. 217, no. 9, pp. 4313-4320, 2011.

[15] A. M. Abd-Alla, S. M. Abo-Dahaba, and R. D. El-Semiry, "Long wavelength peristaltic flow in a tubes with an endoscope subjected to magnetic field," Korea-Australia Rheology Journal, vol. 25, no. 2, pp. 107-118, 2013.

[16] T. Hayat and S. Noreen, "Peristaltic transport of fourth grade fluid with heat transfer and induced magnetic field," Comptes Rendus Mécanique, vol. 338, no. 9, pp. 518-528, 2010.

[17] S. Nadeem and N. S. Akbar, "Influence of radially varying MHD on the peristaltic flow in an annulus with heat and mass transfer," Journal of the Taiwan Institute of Chemical Engineers, vol. 41, no. 3, pp. 286-294, 2010.

[18] S. Nadeem and N. S. Akbar, "Influence of heat transfer on a peristaltic flow of Johnson Segalman fluid in a non uniform tube," International Communications in Heat and Mass Transfer, vol. 36, no. 10, pp. 1050-1059, 2009.

[19] S. Nadeem, T. Hayat, N. S. Akbar, and M. Y. Malik, "On the influence of heat transfer in peristalsis with variable viscosity," International Journal of Heat and Mass Transfer, vol. 52, no. 2122, pp. 4722-4730, 2009.

[20] S. Srinivas, R. Gayathri, and M. Kothandapani, “The influence of slip conditions, wall properties and heat transfer on MHD peristaltic transport," Computer Physics Communications, vol. 180, no. 11, pp. 2115-2122, 2009.

[21] A. M. Abd-Alla, G. A. Yahya, S. R. Mahmoud, and H. S. Alosaimi, "Effect of the rotation, magnetic field and initial stress on peristaltic motion of micropolar fluid," Meccanica, vol. 47, no. 6, pp. 1455-1465, 2012.

[22] S. R. Mahmoud, A. M. Abd-Alla, and M. A. El-Sheikh, "Effect of the rotation on wave motion through cylindrical bore in a micropolar porous medium," International Journal of Modern Physics B, vol. 25, no. 20, pp. 2713-2728, 2011.

[23] A. M. Abd-Alla, S. M. Abo-Dahab, and S. R. Mahmoud, "Wave propagation modeling in cylindrical human long wet bones with cavity," Meccanica, vol. 46, no. 6, pp. 1413-1428, 2011.

[24] S. Akram and S. Nadeem, "Influence of induced magnetic field and heat transfer on the peristaltic motion of a Jeffrey fluid in an asymmetric channel: closed form solutions," Journal of Magnetism and Magnetic Materials, vol. 328, pp. 11-20, 2013.

[25] V. P. Rathod and M. Mahadev, "Peristaltic flow of Jeffrey fluid with slip effects in an inclined channel," Journal of Chemical, Biological and Physical Sciences, vol. 2, no. 4, pp. 1987-1997, 2012.
[26] S. N. Reddy and G. V. Reddy, "Slip effects on the peristaltic pumping of a Jeffrey fluid through a porous medium in inclined asymmetric chanel," International Journal of Mathematical Archive, vol. 4, no. 4, pp. 183-196, 2013. 


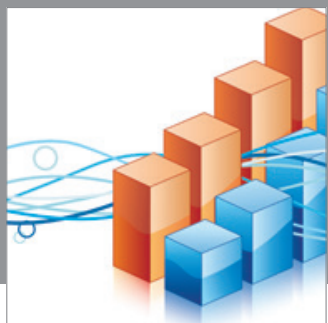

Advances in

Operations Research

mansans

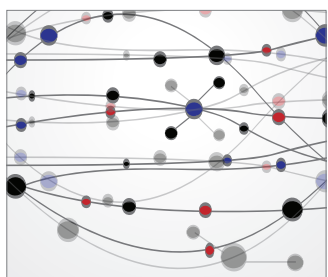

The Scientific World Journal
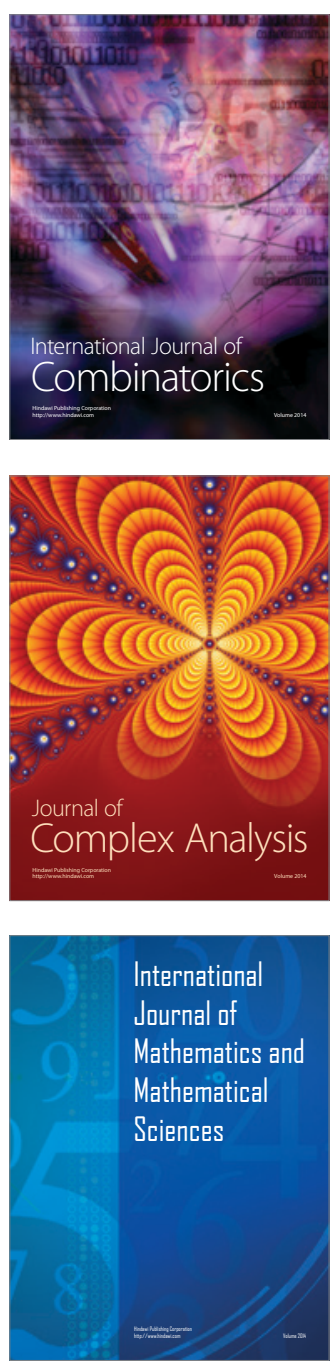
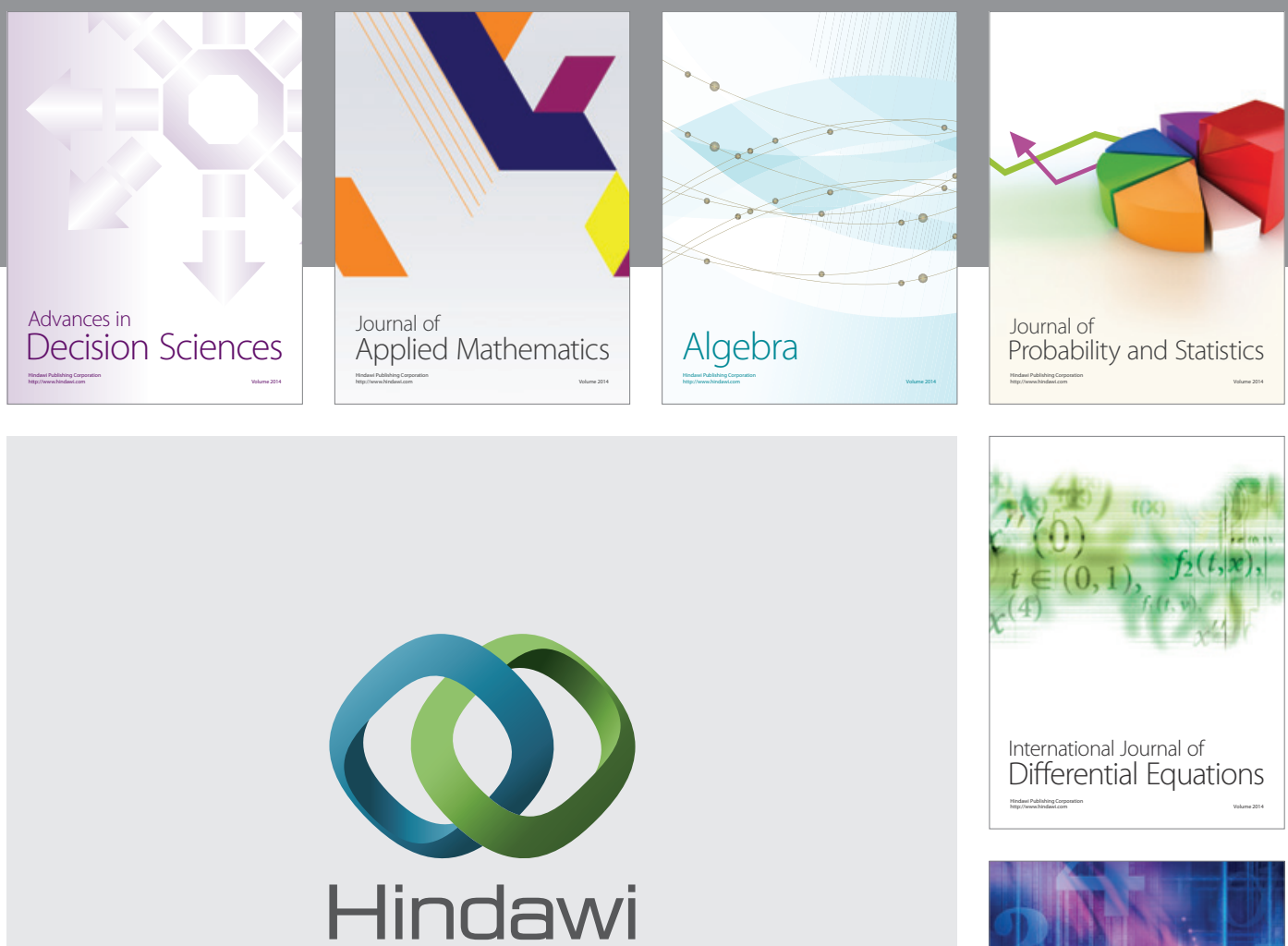

Submit your manuscripts at http://www.hindawi.com
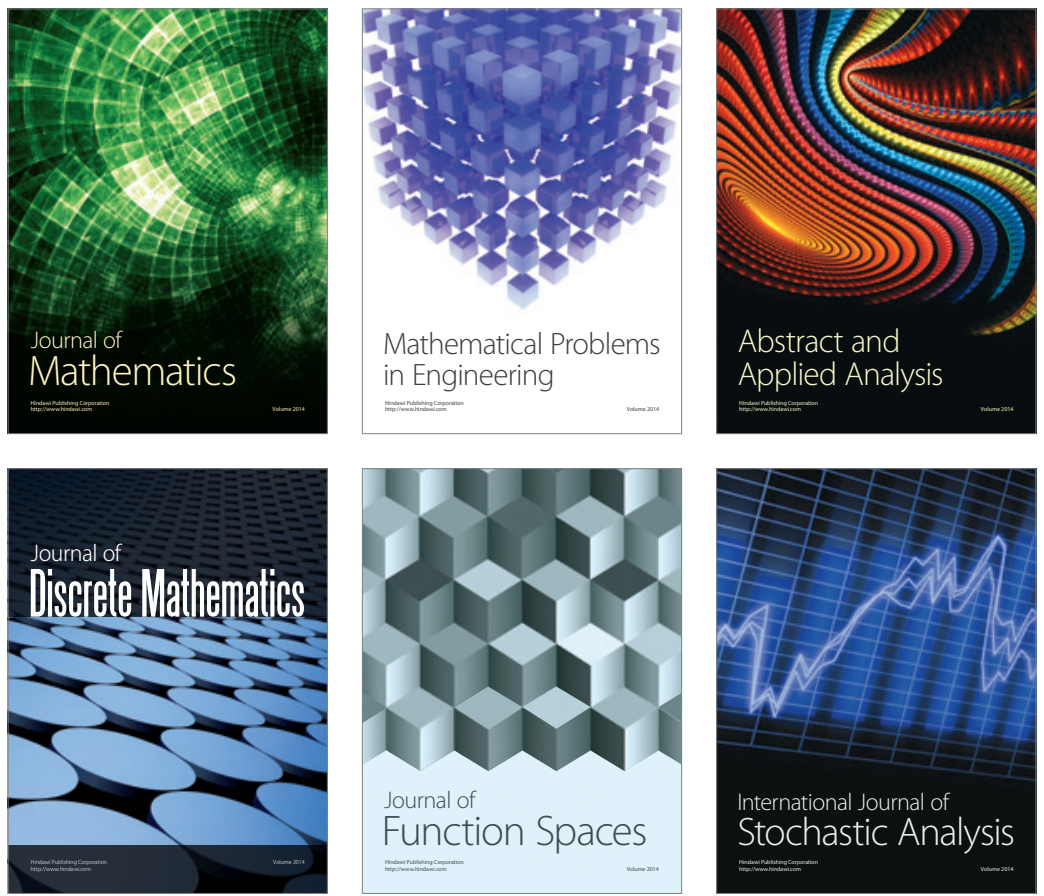

Journal of

Function Spaces

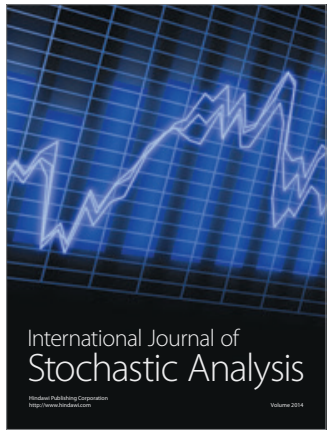

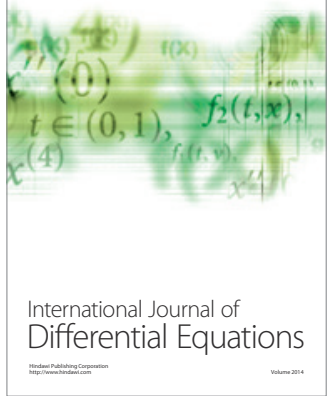
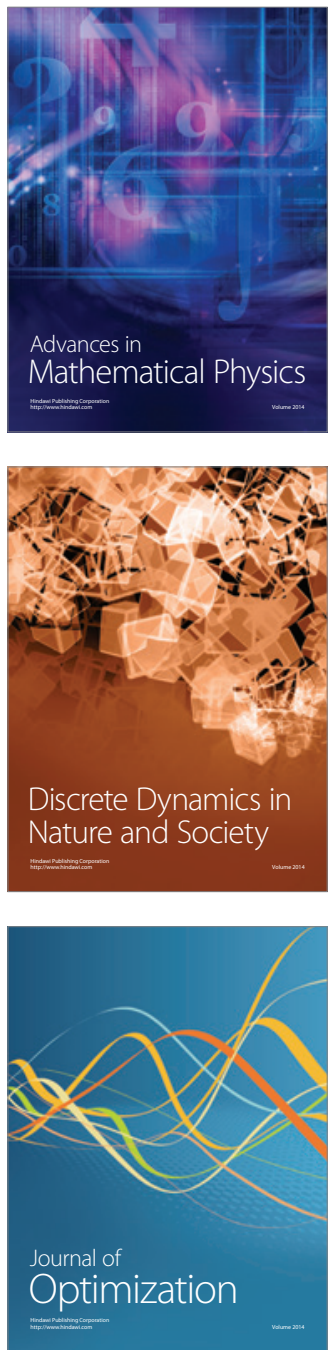